\title{
Antiplatelet therapy in the primary prevention of cardiovascular disease in patients with chronic obstructive pulmonary disease: a randomised controlled proof-of-concept trial
}

\author{
Vijay Kunadian ${ }^{1,2}$, Nina Wilson ${ }^{3}$, Deborah D. Stocken $^{3,4}$, Hani Ali $^{1,2}$, \\ Elaine McColl ${ }^{3}$, Graham Burns $\mathbb{1}^{5}$, Nicola Howe ${ }^{6}$, Andrew Fisher ${ }^{1,2}$ and \\ Anthony De Soyza (10 ${ }^{1,2}$ on behalf of the APPLE COPD-ICON2 trial investigators
}

Affiliations: ${ }^{1}$ Institute of Cellular Medicine, Newcastle University, Newcastle upon Tyne, UK. ${ }^{2}$ Cardiothoracic Centre, Freeman Hospital, Newcastle upon Tyne NHS Foundation Trust, Newcastle upon Tyne, UK. ${ }^{3}$ Institute of Health and Society, Newcastle University, Newcastle upon Tyne, UK. 'Leeds Institute of Clinical Trial Research, University of Leeds, Leeds, UK. ${ }^{5}$ Royal Victoria Infirmary, Newcastle upon Tyne NHS Foundation Trust, Newcastle upon Tyne, UK. ${ }^{6}$ Newcastle Clinical Trials Unit, Newcastle upon Tyne NHS Foundation Trust, Newcastle upon Tyne, UK.

Correspondence: Vijay Kunadian, Institute of Cellular Medicine, Faculty of Medical Sciences, Newcastle University, 4th Floor, William Leech Building, Newcastle-upon-Tyne, NE2 4HH, UK. E-mail: vijay.kunadiandnewcastle.ac.uk

ABSTRACT The APPLE COPD-ICON2 trial is a prospective $2 \times 2$ factorial, double-blinded proof-of-concept randomised controlled trial targeting patients with chronic obstructive pulmonary disease (COPD) without prior history of cardiovascular disease. The primary goal of this trial is to investigate if treatment with antiplatelet therapy will produce the predefined cut-off of platelet inhibition measured using the Multiplate test in COPD patients.

Eligible patients were randomised to aspirin plus placebo, ticagrelor plus placebo, aspirin plus ticagrelor or placebo only for 6 months. The primary outcome comprises inhibition (binary response) of arachidonic acid- (ASPI test, cut-off <40) and adenosine diphosphate- (ADP test, cut-off <46) induced platelet aggregation at 6 months.

543 patients were screened and 120 patients were recruited with mean age of 67.5 years; $47.5 \%$ patients were male. The per-protocol ASPI test response rate to aspirin was $68.3 \%$ (95\% CI 52.3-80.9\%). The perprotocol ADP test response rate to ticagrelaor was 68.8\% (95\% CI 50.4-82.6\%).

Platelet response to antiplatelet therapy with aspirin and ticagrelor was not observed in nearly one-third of COPD patients without prior history of cardiovascular disease. These findings support the high prothrombotic milieu and the need for further research to determine the effect of antiplatelet/antithrombotic therapy on cardiovascular morbidity and mortality in COPD patients.

@ERSpublications

COPD is the world's number 2 killer. In our study, treatment with antiplatelet therapy in COPD patients did not lead to adequate platelet response in just under a third of patients, emphasising the high thrombotic milieu in these patients. http://bit.ly/2WT8241

Cite this article as: Kunadian V, Wilson N, Stocken DD, et al. Antiplatelet therapy in the primary prevention of cardiovascular disease in patients with chronic obstructive pulmonary disease: a randomised controlled proof-of-concept trial. ERJ Open Res 2019; 5: 00110-2019 [https://doi.org/ 10.1183/23120541.00110-2019].

This article has supplementary material available from openres.ersjournals.com

This study is registered at EudraCT with identifier number 2014-005475-86, the UK Clinical Research Network with identifier 19244 and ISCTRN with identifier ISRCTN43245574. The authors are very happy to share data.

Received: May 082019 | Accepted after revision: June 112019

Copyright $\odot$ ERS 2019. This article is open access and distributed under the terms of the Creative Commons Attribution Non-Commercial Licence 4.0. 


\section{Introduction}

Chronic obstructive pulmonary disease (COPD) is a significant global health burden [1] and is in the world's top three global killers. The major cause of COPD is smoking and there is an excess of cardiovascular disease seen in COPD patients as compared to those matched for smoking exposure without COPD. The prevalence of coronary artery disease (CAD) in COPD patients has been estimated at 10-38\% [2,3] with 20-50\% of mortality in COPD being related to cardiovascular causes [4,6-] The ECLIPSE (Evaluation of COPD Longitudinally to Identify Predictive Surrogate Endpoints) observational cohort study showed that the presence of CAD in patients with COPD is associated with poor clinical outcomes [7].

Several mechanisms have been proposed to explain the increased risk of CAD in COPD patients. Spill-over of pulmonary inflammation to the systemic circulation has been proposed as a possible mechanism. Another proposed mechanism contributing to CAD in COPD is an increased in vivo platelet activation state, which plays a key role in the pathogenesis of atherothrombosis. Monocyte-platelet aggregates, a marker of platelet activation, are elevated in both patients with stable and acute exacerbation of COPD [8].

The use of aspirin in the primary prevention among individuals without cardiovascular disease has been shown to be associated with a lower risk of cardiovascular events and an increased risk of major bleeding [9]. Ticagrelor has been used in primary prevention among type 2 diabetic patients in the THEMIS (The Effect of Ticagrelor on Health Outcomes in Diabetes Mellitus Patients Intervention Study) trial [10]. Preliminary results showed that ticagrelor taken in conjunction with aspirin showed a statistically significant reduction in a composite of major adverse cardiovascular events compared to aspirin alone. The role of antiplatelet therapy (APT) using aspirin and ticagrelor in the primary prevention of cardiovascular disease among COPD patients has not been evaluated previously. The aim of this early-phase trial is to evaluate the effect of antiplatelet therapy (aspirin, ticagrelor) on platelet function and explore its effect on the inflammatory markers in patients with COPD with no prior history of CAD.

\section{Methods}

\section{Trial design}

The APPLE COPD trial is a single-centre, early-phase, $2 \times 2$ factorial, double-blind randomised controlled trial. Investigators/assessors and patients were blinded through the use of matched placebo tablets. The early-phase design allows decision-making regarding the further investigation of either aspirin or ticagrelor in late-phase trials. Full details of the trial design and analysis plan have been published previously [11]

Eligibility criteria specified that patients were aged $\geqslant 18$ years, with $\geqslant 10$ pack-years smoking history, confirmed airflow limitation on spirometry (forced expiratory volume in $1 \mathrm{~s}$ (FEV1) $<80 \%$ predicted and $\mathrm{FEV}_{1} /$ forced vital capacity (FVC) ratio 0.7 ) and had given written informed consent. Exclusion criteria for the study included any condition concurrently treated through anticoagulation or APT, including aspirin or ticagrelor; specific contraindications to management with antiplatelet medication; or aspirin and ticagrelor use. The trial was carried out in accordance with the Declaration of Helsinki. The regional National Health Service (NHS) research ethics committee (REC15/NE/0155) gave approval for this trial.

\section{Trial interventions}

Consenting patients were randomised using the Newcastle Clinical Trials Unit secure web-based system to ensure concealment of allocation, and allocated in a double-blind manner into one of four groups (aspirin plus placebo, ticagrelor plus placebo, aspirin plus ticagrelor (dual antiplatelet therapy (DAPT)) or placebo only) using permuted random blocks of variable length, stratified by age ( $\leqslant 65$ years, $>65$ years). Aspirin $(75 \mathrm{mg})$ was administered once daily dose and ticagrelor $(90 \mathrm{mg})$ twice daily for a 6-month period, provided as two 3-month supplies. Compliance to medication was assessed at 1 month, 3 months and 6 months by the research team members in the research clinic.

\section{Outcome measures}

The primary outcome measure consisted of inhibition (binary response, yes/no) of the ASPI test with a responder cut-off $<40$ for patients randomised to aspirin, and the adenosine diphosphate (ADP) test with a responder cut-off $<46$ for patients randomised to ticagrelor. Platelet aggregation induced by ADP (ADP test) or arachidonic acid (ASPI test) was assessed by whole-blood impedance aggregometry using the Multiplate device (Roche Diagnostics, Burgess Hill, UK). The ASPI test is sensitive to an inhibition of the platelet cyclo-oxygenase, which is the target of aspirin, and the ADP test is sensitive to inhibition of $\mathrm{P}_{2} \mathrm{Y}_{12}$, which is the target of ticagrelor.

Secondary outcome measures were changes in systemic inflammatory markers including fibrinogen, high-sensitivity C-reactive protein (hsCRP), tumour necrosis factor (TNF)- $\alpha$, interleukin (IL)-6, myeloperoxidase (MPO) from baseline to 6 months; changes in carotid intima media thickness and non-invasive measures of vascular stiffness (pulse wave velocity) from baseline to 6 months; and changes 
in quality of life at baseline and at 6 months were evaluated using questionnaires (Euroqol five dimensions, five levels (EQ5D-5L), St George's Respiratory Questionnaire for COPD Patients (SGRQ-C)). In addition, we recorded subjective reports of breathlessness using the Medical Research Council Dyspnoea (MRCD) scale. The primary safety measure was the rates of major and minor bleeding as defined by the BARC (Bleeding Academic Research Consortium) definition [12]. In addition, given that ticagrelor is associated with dyspnoea, assumed to be due to its adenosine-mediated effects, we monitored FEV1, FVC and MRCD scale as a safety outcome measure. All non-serious adverse reactions were recorded at 6-month follow-up, and serious adverse events were recorded throughout the duration of the trial until 4 weeks after trial therapy was stopped.

\section{Statistical considerations}

A Fleming-A'Hern [13] early-phase design was used to investigate the roles of each intervention (aspirin, ticagrelor) in achieving a minimum level of response at 6 months worthy of further investigation. PERL et al. [14] reported a response rate for ticagrelor and prasugrel at 1 month of $100 \%$ and $91.3 \%$, respectively in a non-randomised comparison in patients with myocardial infarction. APPLE COPD assumed a response rate to reject either treatment $(\mathrm{p} 0)<65 \%$ and a response rate to investigate either treatment further $(\mathrm{p} 1)>80 \%$. The justification to investigate either treatment further is based on observing a critical number of responses for each intervention, specifically 44 for aspirin and 42 for ticagrelor according to the Fleming-A'Hern design. The control arm provides a direct and unbiased benchmark.

With error levels of $12 \% \alpha$ (type 1) and $12 \% \beta$ (type 2), inflated due to the early-phase nature of the design, the target recruitment was 50 patients per arm for each comparison within the $2 \times 2$ design, namely 1) aspirin versus no aspirin; 2) ticagrelor versus no ticagrelor. The sample size was adjusted for an anticipated 15\% drop-out rate within the 6-month timeframe to a target recruitment of 120 patients.

As a $2 \times 2$ factorial design, the primary analyses are based on a comparison of aspirin versus no aspirin and ticagrelor versus no ticagrelor. Response rate was calculated on an intention to treat (ITT) basis as the total number of patients responding at 6 months as a proportion of all patients randomised, reported as an unadjusted odds ratio with $95 \%$ confidence intervals. A preplanned sensitivity analysis was carried out on the per-protocol set removing patients who have withdrawn completely, were lost to follow-up or who discontinued treatment. Adverse events are reported descriptively in terms of the number of events and number of unique patients experiencing an adverse event expressed as a proportion of the total number of patients starting treatment. Descriptive data are presented for demographics and baseline characteristics.

\section{Results}

\section{Baseline characteristics}

120 patients were recruited. The CONSORT (Consolidated Standards of Reporting Trials) diagram is shown in figure 1. Patient characteristics were balanced across arms (table 1 and supplementary table S1). The mean age of patients was 67.5 years and 57 (47.5\%) patients were male. The median QRISK2 score was 22.0 (interquartile range (IQR) 15.5-29.0). Of the 117 patients with current COPD medication information, $88(75.2 \%)$ were on triple inhaled therapy (inhaled corticosteroid (ICS)/long-acting $\beta$-agonist (LABA)/long-acting muscarinic antagonist), 12 (10.3\%) were on ICS/LABA, 11 (9.4\%) were on LABA alone, nine $(7.7 \%)$ were on long-term azithromycin and four $(3.4 \%)$ were on long-term oxygen. The laboratory tests and other study related variables are shown in table 2 and supplementary table S2.

\section{Treatment received}

All 120 randomised patients started treatment. The median (IQR) time on treatment from randomisation across all arms was 182 (175-189) days (supplementary table S3). Treatment compliance was high, with $91 \%$ and $88 \%$ of the 102 participants who reached 6 months taking $\geqslant 80 \%$ of the protocol dose for aspirin/ placebo aspirin and ticagrelor/placebo ticagrelor, respectively (supplementary table S4).

\section{Primary outcome measure}

The ITT and per-protocol analysis of the primary outcome measure is shown in table 3 . In the 60 patients randomised to aspirin, at 6 months, there were 29 (48.3\%, 95\% CI 35.8-61.0\%) ASPI responders observed in the ITT analysis. This rate is lower than anticipated in the statistical design and did not reach the critical threshold of 44 responders required to warrant further research, according to the Fleming-A'Hern design. In the 58 patients randomised to ticagrelor, there were 24 (41.4\%, 95\% CI 29.3-54.6\%) ADP responders observed in the ITT analysis. This rate is lower than anticipated in the design and did not reach the critical threshold of 42 responders required to warrant further research. Figure $2 \mathrm{a}$ shows a reduction in ASPI from baseline in the aspirin group at 1 month and 6 months, but no reduction in the no aspirin group. There was more variation in the aspirin group at 6 months as shown by the IQR. A reduction in ADP is observed in the ticagrelor group at 1 and 6 months compared to baseline. This 


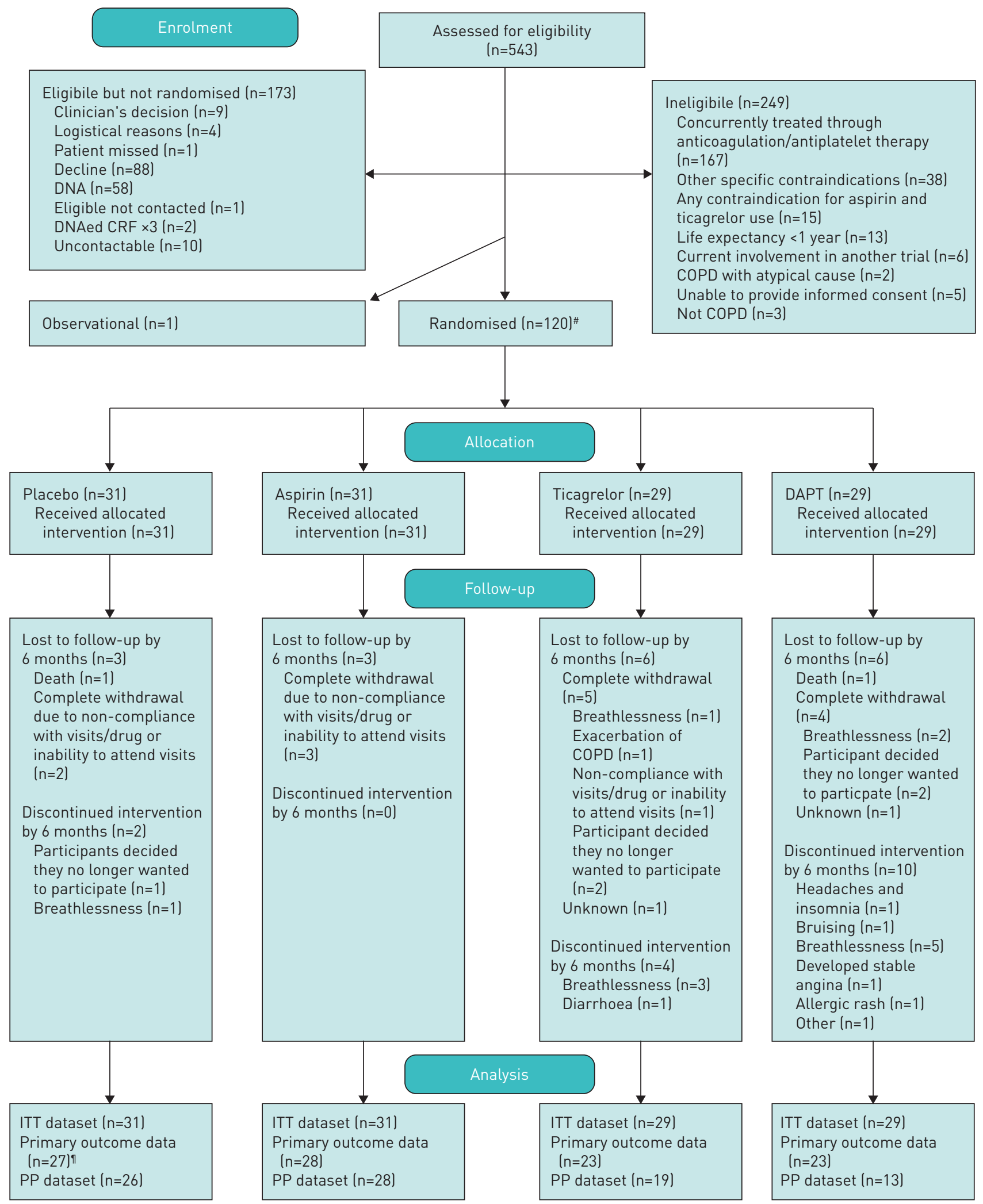

FIGURE 1 CONSORT (Consolidated Standards of Reporting Trials) diagram. COPD: chronic obstructive pulmonary disease; DAPT: dual antiplatelet therapy; ITT: intention-to-treat; PP: per-protocol. ${ }^{\#}$ : there was one administrative error, so there were 121 randomisations, of which 120 were related to patients; ${ }^{\text {ๆ }}$ : one patient attended visit but the lab samples were not suitable for analysis. 


\section{TABLE 1 Baseline characteristics}

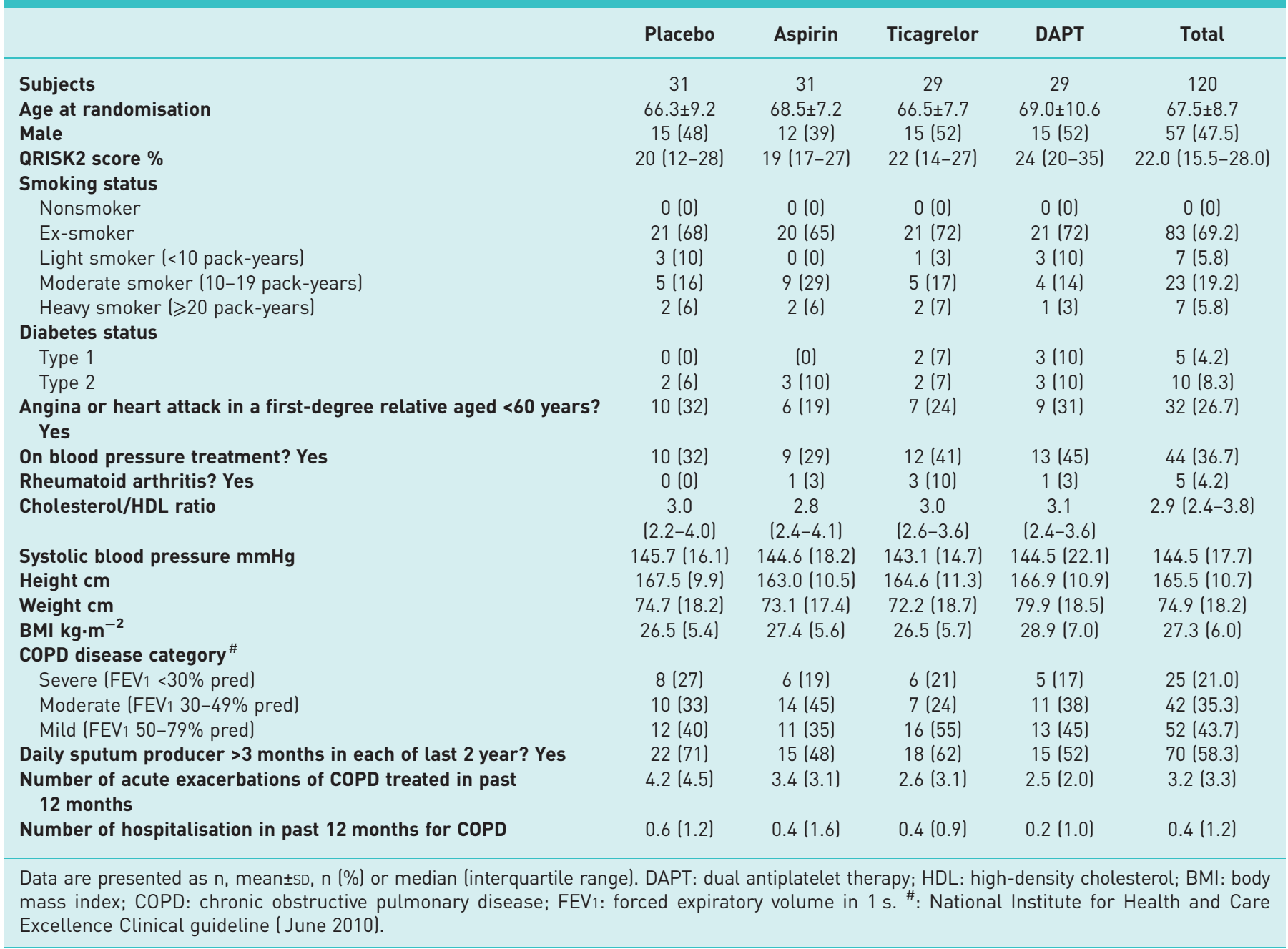

reduction was not seen in the no-ticagrelor group. There is larger variation in the ADP score at 6 months in the ticagrelor group (figure $2 b$ ).

As a prespecified sensitivity analysis, we calculated the rate of response on a per-protocol analysis set consisting of $86(72 \%)$ of the 120 randomised participants (figure 1). In the aspirin group there were 28 (68.3\%, 95\% CI 52.3-80.9\%) ASPI responders observed (table 3). In the ticagrelor group there were 22 (68.8\%, 95\% CI 50.4-82.6\%) ADP responders observed. The larger variation in response at 6 months compared to 1 month is reduced for both the aspirin and ticagrelor groups when the data is summarised on the per-protocol analysis set (figure 3). Supplementary figure S1 shows that the larger variability present in the aspirin and ticagrelor groups is from the DAPT arm.

\section{Secondary outcome measures}

There were no observed differences between the groups in the changes in quality of life using questionnaires (EQ5D-5L, SGRQ-C) from baseline to 6 months (figures 4 and 5, respectively). There were no clinically significant differences in changes in inflammatory markers including fibrinogen, hsCRP, TNF- $\alpha$, IL- 6 and MPO from baseline to 6 months and there were no clinically significant differences in changes in carotid intima media thickness and non-invasive measures of vascular stiffness from baseline to 6 months in the different groups (supplementary figures S2, S3, S4).

\section{Safety outcomes}

Five type 1 bleeds according to the BARC criteria were recorded in this study: two in the placebo arm, two in the aspirin arm and one in the ticagrelor arm. They were all categorised as minimal on the Thrombolysis in Myocardial Infarction scale (supplementary table S5). Dyspnoea was measured using the 
TABLE 2 Laboratory and other secondary outcomes data

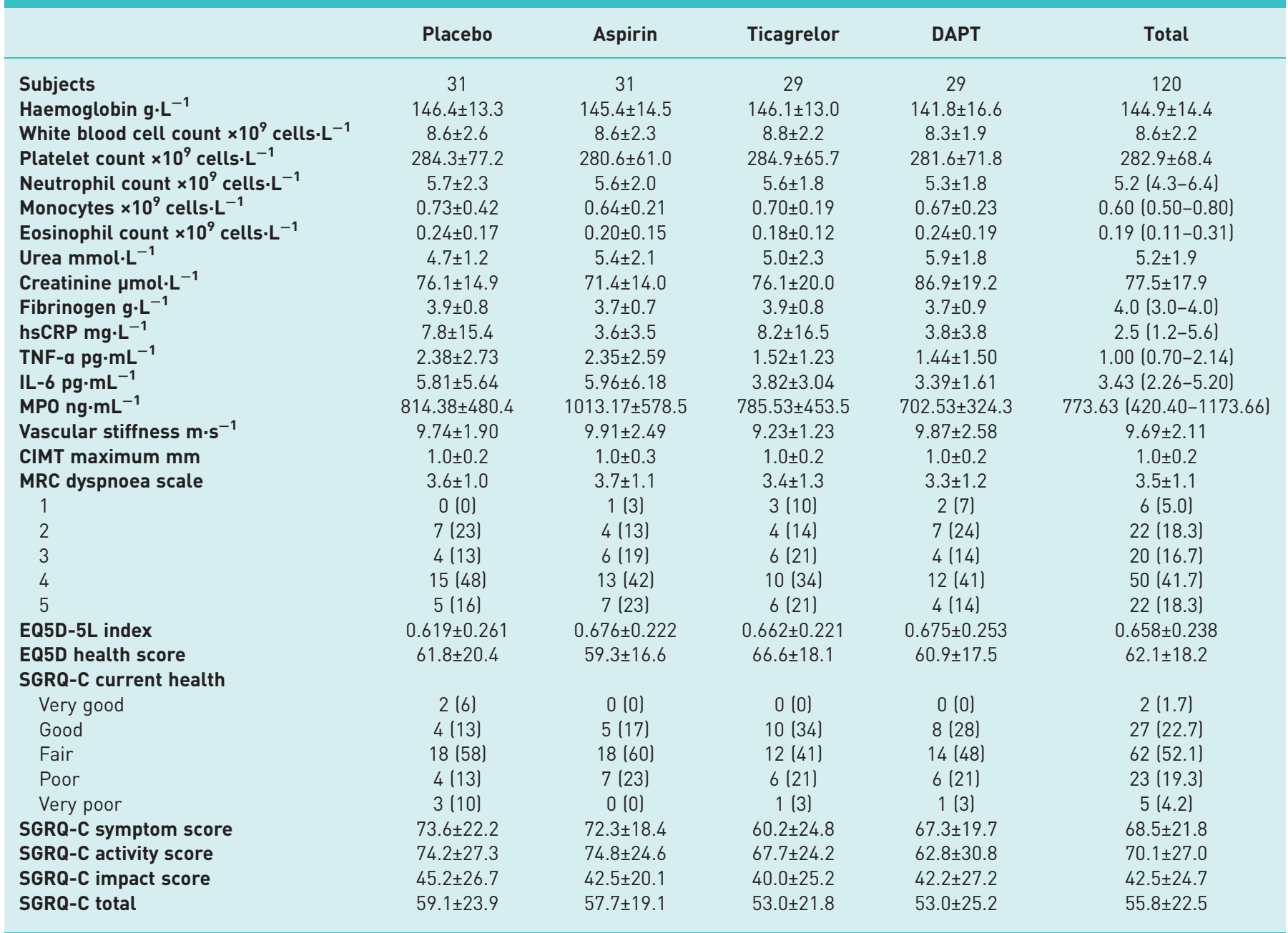

Data are presented as $\mathrm{n}$, mean $\pm \mathrm{SD}$, median (interquartile range) or $\mathrm{n}(\%)$. DAPT: dual antiplatelet therapy; hsCRP: high-sensitivity C-reactive protein; TNF: tumour necrosis factor; IL: interleukin; MPO: myeloperoxidase; CIMT: carotid intima media thickness; MRC: Medical Research Council; SGQR-C: St George's Respiratory Questionnaire for COPD patients.

MRCD scale, where a score of 1 equates to minimal breathlessness and 5 equates to housebound due to breathlessness. The MRCD score was similar across the groups and at all time points. This was also the case for FEV1 and FVC (supplementary table S6).

Adverse events and serious adverse events

The adverse events are shown in supplementary table S7. During the study, one participant, who was in the DAPT arm, experienced a severe adverse event that was considered to be definitely treatment related. This adverse event was breathlessness and the treatment was discontinued. There were 31 serious adverse events in 10 patients $(8 \%, n=120)$, none of which were considered to be related to treatment (supplementary table S8).

\section{Discussion}

Increasingly it is accepted that COPD is a complex multisystems disorder [15]. In addition to neuropsychiatric morbidity, metabolic syndrome, obesity and diabetes, COPD has been shown to be linked with systemic inflammation, thrombocytosis and activation of prothrombotic pathways [16]. The end result of these is an elevated risk of CAD. However, to date there are few studies specifically targeting the excess CAD risk in COPD patients. Cardiovascular complications in COPD are one of the most costly comorbidities [17].

Our data are novel in that they demonstrate in the per-protocol analysis that just over two-thirds of patients with COPD demonstrated platelet response to antiplatelet therapies compared to $100 \%$ of patients 


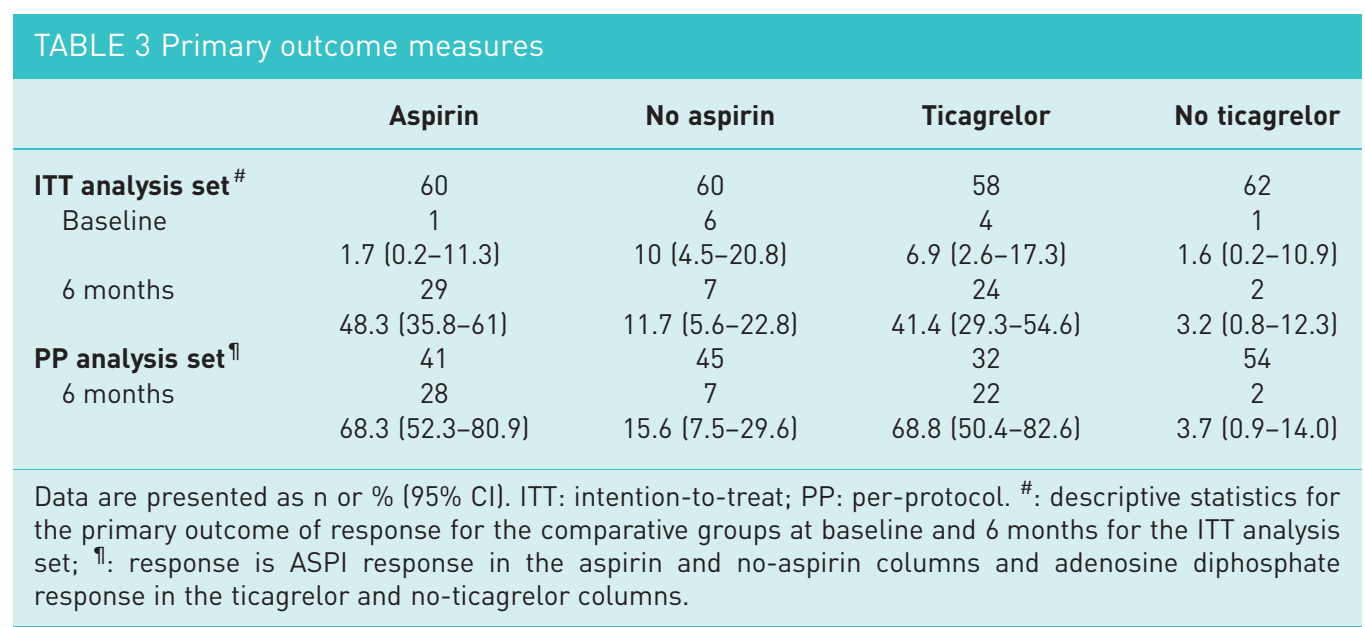

with myocardial infarction [14]. Interestingly, we noted that for both ASPI and ADP tests $>30 \%$ of patients in the per-protocol analysis did not demonstrate platelet response to therapeutic targeting based on recognised thresholds in the primary prevention setting in our population. High on-treatment platelet reactivity despite use of $\mathrm{P}_{2} \mathrm{Y}_{12}$ antagonists is associated with adverse cardiac events [18]. A previous study in the setting of secondary prevention showed that COPD patients who underwent percutaneous coronary intervention have higher "on treatment" platelet reactivity compared to non-COPD patients and suggested that this could contribute to the high cardiovascular mortality in these patients [19]. Reassuringly, our data demonstrate that there were mean response rates in ADP and ASPI tests that were very similar at 1 month and 6 months, suggesting that the effects were sustained.

We noted more withdrawals in those treated with ticagrelor than those treated with aspirin alone or placebo. Despite the significant airflow limitation seen in this group only one patient had an adverse event reported as severe breathlessness requiring drug discontinuation. The withdrawal rates were higher in the
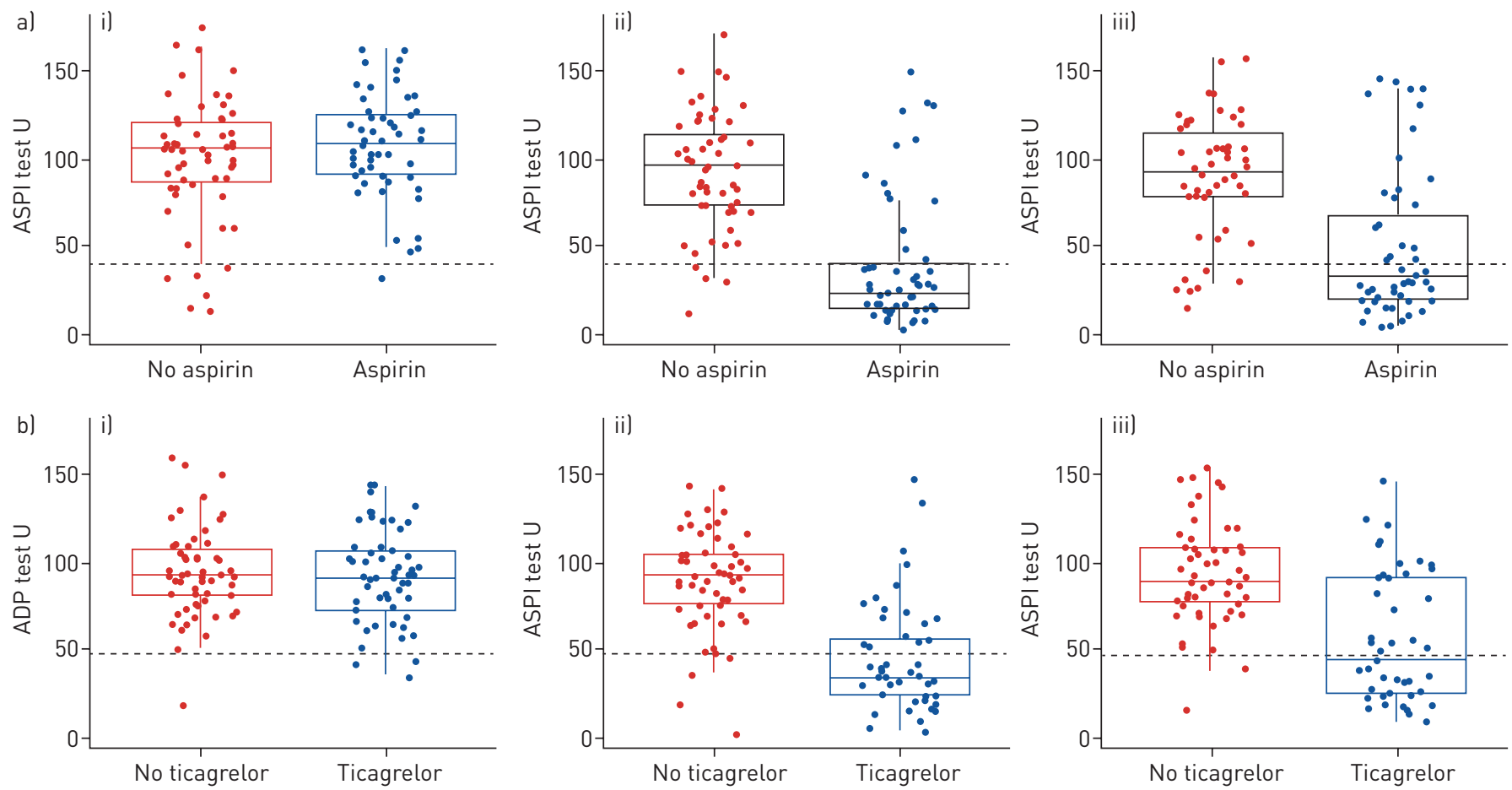

FIGURE 2 Primary outcome measure intention-to-treat (ITT) analysis. Boxplots of the a) ASPI test split by no-aspirin and aspirin comparative groups over time; b) adenosine diphosphate (ADP) test split by no-ticagrelor and ticagrelor comparative groups over time (ITT analysis set). i) Baseline; ii) 1 month; iii) 6 months. The green dashed line indicates the threshold below which patients are considered to be responders. 


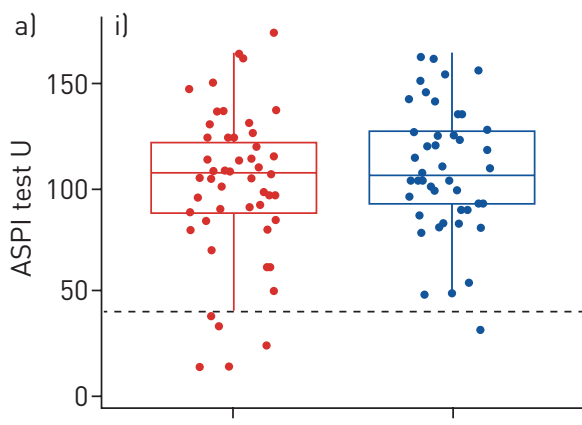

No aspirin

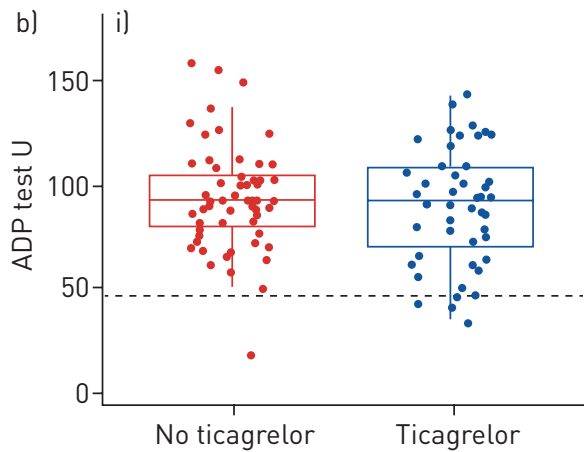

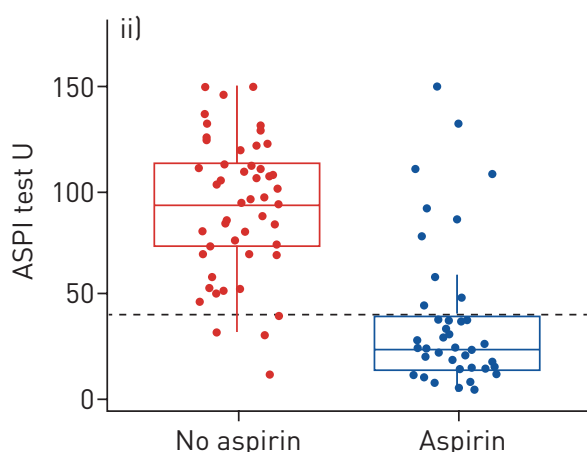
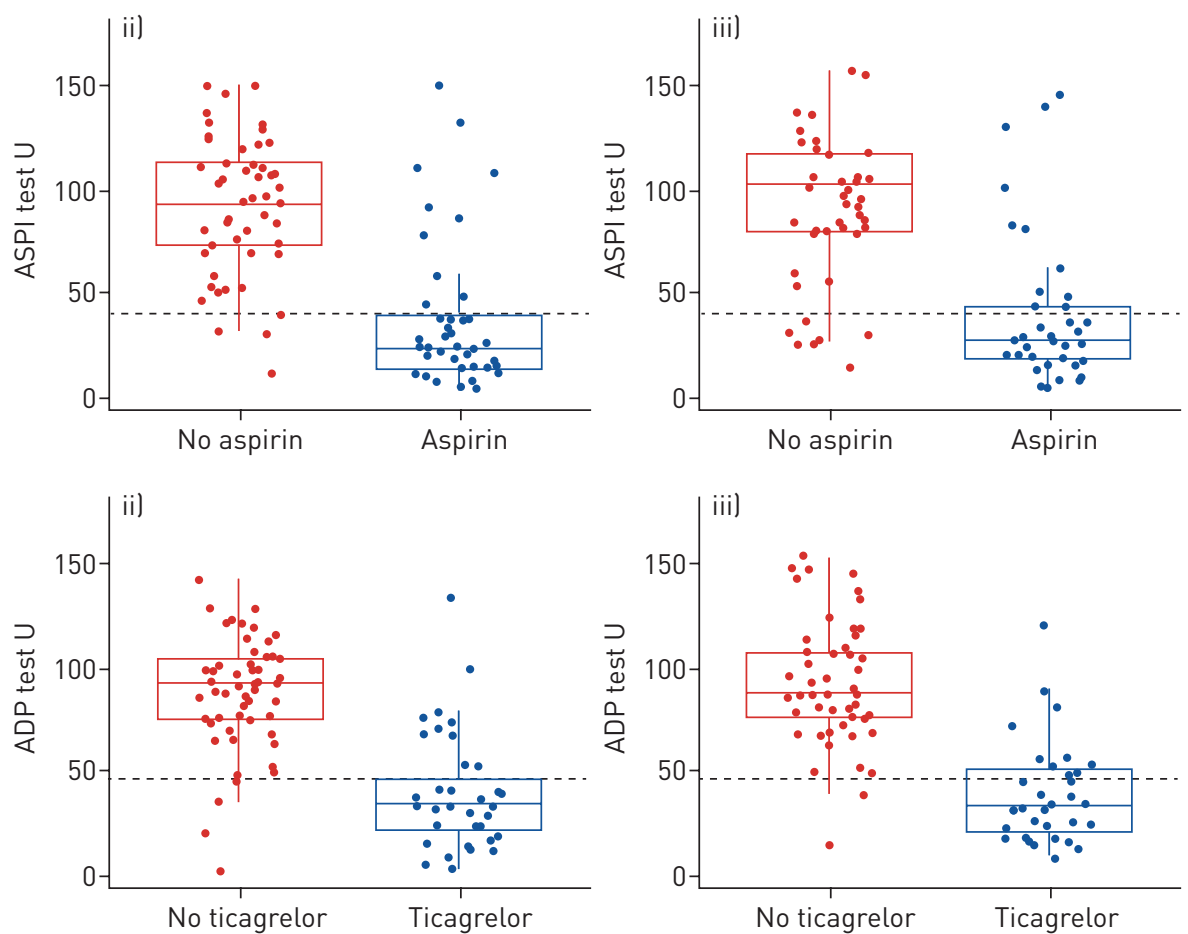

FIGURE 3 Primary outcome measure per-protocol analysis. Boxplots of the a) ASPI test split by no-aspirin and aspirin comparative groups over time; b) adenosine diphosphate (ADP) test split by no-ticagrelor and ticagrelor comparative groups over time with patients who withdrew, discontinued treatment or lost to follow-up by the timepoint of interest removed (per-protocol analysis set). i) Baseline; ii) 1 month; iii) 6 months. The green dashed line indicates the threshold below which patients are considered to be responders.
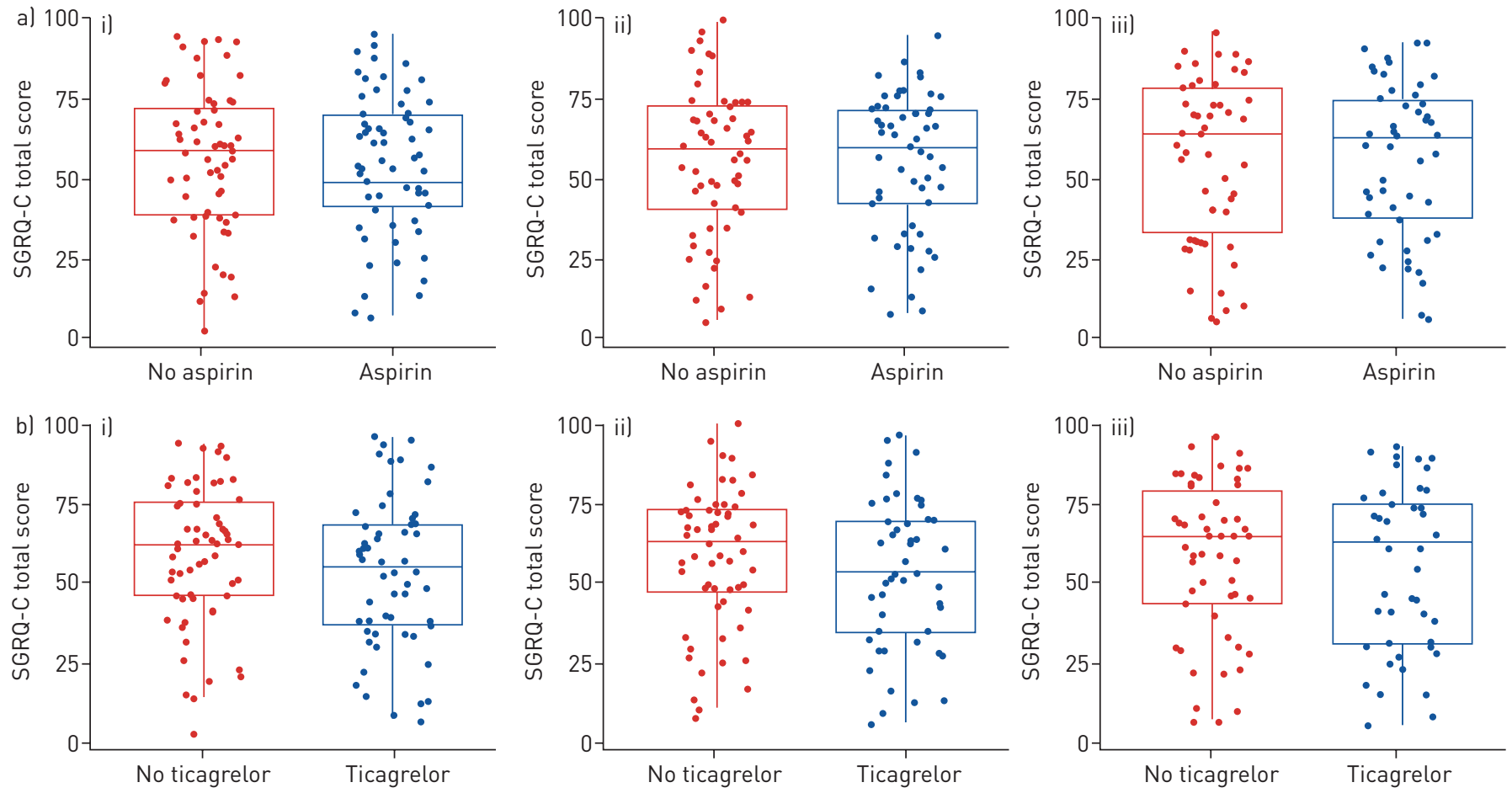

FIGURE 4 Secondary outcome measure St George's Respiratory Questionnaire for chronic obstructive pulmonary disease patients (SGRQ-C). Boxplots of SGRQ-C total score over time by comparative groups split by a) no aspirin and aspirin and b) no ticagrelor and ticagrelor (intention-to-treat analysis set). i) Baseline; ii) 1 month; iii) 6 months. 

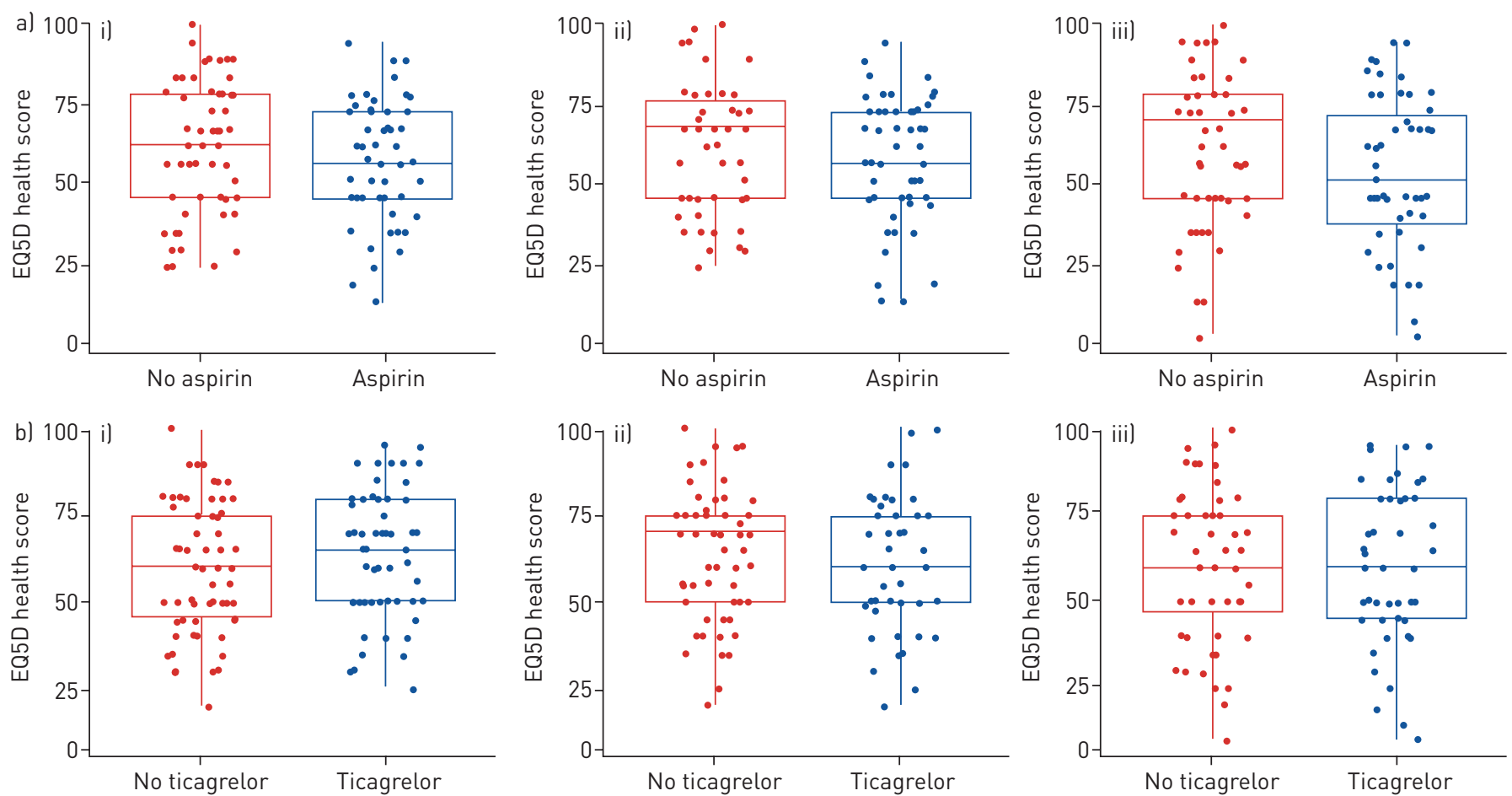

FIGURE 5 Secondary outcome measure Euroqol five-dimensions (EQ5D) health score over time by comparative groups split by a) no aspirin and aspirin and b) no ticagrelor and ticagrelor (intention-to-treat analysis set). i) Baseline; ii) 1 month; iii) 6 months.

ticagrelor arms, but were similar to those reported in a previous study of ticagrelor where nearly $11 \%$ of patients stopped therapy due to dyspnoea [20]. Nevertheless, we observed reduced platelet response in patients receiving aspirin as well as those receiving ticagrelor, suggesting that this is a true reflection of response not merely due to drug compliance in our COPD patients. Importantly, our further per-protocol analysis (excluding patients who have withdrawn completely, were lost to follow-up or who discontinued treatment) showed that $>30 \%$ of patients did not demonstrate the required platelet response to antiplatelet therapy.

Several commercially available validated tests are used to evaluate platelet response to therapy. In our study we used the Multiplate test, which has previously been shown to be concordant with VerifyNow and vasodilator stimulated phosphoprotein (VASP) tests. The Multiplate test has been shown to best reflect the in vivo plasma concentrations of ticagrelor and its active metabolite compared to other tests (VerifyNow and VASP) [21]. Other studies have shown Multiplate, VASP and platelet function analyser tests to have moderate agreement regarding the classification of patients as responders to $\mathrm{P}_{2} \mathrm{Y}_{12}$ inhibition [22]. Therefore, the Multiplate test is a valid method of evaluating platelet response to antiplatelet therapy in our patient cohort. Of note, platelet reactivity values have not been shown to be predictive of cardiovascular morbidity and mortality [23]. Our study was not designed to evaluate cardiovascular morbidity and mortality.

One of the key concerns in initiating antiplatelet therapy in primary prevention is bleeding. We specifically sought known side-effects of antiplatelet therapy such as bleeding (aspirin and ticagrelor) and dyspnoea (ticagrelor). In our study we did not identify any clinically significant bleeding in patients receiving antiplatelet therapy. This might be due to the fact that our study had small number of patients and were treated with antiplatelet therapy for 6 months. Previous studies have shown that ticagrelor-related dyspnoea is usually mild or moderate in intensity and does not appear to be associated with differences concerning any efficacy or safety outcomes with ticagrelor compared with clopidogrel therapy [24]. In our study we did not observe clinically significant dyspnoea in our patient cohort. These findings support the safety of using antiplatelet therapy in the primary prevention in patients with COPD. In a previous post hoc analysis, COPD patients experienced high rates of ischaemic events compared to non-COPD patients. Ticagrelor versus clopidogrel substantially decreased the absolute risk of ischaemic events (5.8\%) in COPD patients, without increasing overall major bleeding events [25]. Based on previous studies, we anticipated a close to $100 \%$ platelet response to ticagrelor in our patient cohort. However, this was not demonstrated in our study. These findings support the high prothrombotic milieu in COPD patients, where in addition to 
potent antiplatelet therapy, an antithrombotic therapy might be beneficial in addressing the excess cardiovascular morbidity and mortality. Of note, although several serious adverse events were recorded in our study, reassuringly none of these events were related to the drugs utilised in our study as such.

There were no improvements or decrements in the quality of life responses on antiplatelet therapy raising the potential for long-term studies. Of note, in a previous study, short-term administration of high doses of ticagrelor did not appear to alter pulmonary function at rest and during exercise in subjects at risk of (healthy elderly) or with respiratory impairment (mild asthma or mild-to-moderate COPD) [26]. Neurohormonal activation and systemic inflammation have been suspected to play a significant role in increasing the risk of cardiovascular disease [27, 29]. Ticagrelor as compared to clopidogrel is superior in improving surrogate markers of endothelial function and on-treatment platelet reactivity [30]. In our study, antiplatelet therapy did not have any clinically significant effect on any of the secondary outcome measures including inflammatory biomarkers.

\section{Strengths and limitations}

The strengths of our study included the recruitment of patients with COPD who were representative of a secondary care cohort with many patients with severe airflow limitation and multiple comorbidities. The average QRISK2 score was $>20 \%$, confirming the fact that increased cardiovascular risk is prevalent in this population.

Our study is limited by small sample size and short duration of antiplatelet therapy. However, although this sample is not powered to address hard end-points, it is sufficiently powered to detect differences in platelet reactivity between the groups. In our study we specifically included patients without prior history of cardiovascular disease. Use of antiplatelet therapy has already been studied in patients with COPD and cardiovascular disease. There is no consensus on the optimal duration to assess antiplatelet therapy when long-term treatment is planned. We chose a 6-month period as a pragmatic time point to ascertain the effects and side effects of more prolonged therapy. Reflecting the chronic nature of COPD, long-term treatment regimens will be needed to derive cardiovascular protection and hence other time points $(\geqslant 12$ months) may have been equally important. Despite randomisation, the biomarker values were not evenly distributed at baseline in our study. Nevertheless, we did not demonstrate any differences in biomarker levels between baseline values and during follow-up in the different groups. This might also be attributed to small sample size in our study.

\section{Conclusion}

Platelet response (the predefined cut-off of platelet inhibition measured using the Multiplate test) to antiplatelet therapy with aspirin and ticagrelor was not observed in nearly one-third of patients in the per-protocol analysis. Bruising is a common side-effect, but clinically significant bleeding is uncommon. Some patients experience dyspnoea on ticagrelor, but this is mild or moderate in the majority of patients. These findings support the high prothrombotic milieu in COPD patients where in addition to potent antiplatelet therapy, an antithrombotic therapy might be beneficial in addressing the excess cardiovascular morbidity and mortality warranting further investigation.

Acknowledgements: We are grateful to Andrew Fisher, Graham Burns, Sophie West, Stephen Bourke and Jim Lordan for allowing their patients to be included in this trial; clinical research fellows Hani Ali and Danny Chan; the primary care team (Laura Renwick and Sally Dunn); all general practices in the North East and Cumbria Primary Care Clinical Research Network that contributed patients; Philip McGrouther, Louise McCormack, Ashley Eglon and Vanessa Ludley (Newcastle NIHR Clinical Research Facility, Newcastle upon Tyne, UK); Matthew Larman and Sven Nylander (AstraZeneca); and Ian Campbell, Maria Allen and Lesley Rigden (Newcastle Hospitals Pharmacy Department, Newcastle upon Tyne, UK).

Trial management group: Vijay Kunadian (chief investigator); Anthony De Soyza (principal investigator); Deborah Stocken (senior statistician and coinvestigator); Andrew Fisher (coInvestigator); Nina Wilson (trial statistician); Graham Burns (investigator); Nicola Howe (NCTU Database Manager); Sean Scott (sponsor representative); Elaine McColl (coinvestigator); Jared Thornton and Lesley Hall (NCTU senior trial managers); Alexander von Wilamowitz-Moellendorff and Andrea Bell (NCTU trial managers); Eva-Maria Holstein (NCTU trial administrator); Laura Robertson (trial secretary); and Cath Brennand and Jaki Begum (NCTU close-down team).

Trial oversight committee: Stephan James (chair; Uppsala Clinical Research Centre, Uppsala University, Sweden), Neil Swanson (James Cook University Hospital, Middlesbrough, UK), Alex McConachie (Institute of Health and Wellbeing, Robertson Centre for Biostatistics, University of Glasgow, UK) and John Hurst (Centre for Inflammation and Tissue Repair, University College London, UK).

Conflict of interest: None declared.

Support statement: The research was supported by an external research grant from AstraZeneca (funder reference number ISSBRIL0303), and the National Institute for Health Research (NIHR) Newcastle Biomedical Research Centre 
based at Newcastle Hospitals NHS Foundation Trust and Newcastle University. The views expressed are those of the author(s) and not necessarily those of the NHS, the NIHR or the Department of Health. Trial sponsor and funder had no role in the design of this study and did not have any role during its execution, analyses, interpretation of the data, or decision to submit results. Statistical analyses were carried out by the trial statisticians. The manuscript was prepared by all authors. The decisions to submit for publication was agreed by all authors. The corresponding author had access to all the data and had final responsibility for the decision to submit for publication, under the authorship agreed with all the coinvestigators and Newcastle Clinical Trials Unit staff who fulfil the International Committee of Medical Journal Editors criteria for authorship. Funding information for this article has been deposited with the Crossref Funder Registry.

\section{References}

1 Global Initiative for Chronic Obstructive Lung Disease (GOLD). 2018. . Global Strategy for the Diagnosis, Management and Prevention of COPD. http://goldcopd.org/.

2 Antonelli Incalzi R, Fuso L, De Rosa M, et al. Co-morbidity contributes to predict mortality of patients with chronic obstructive pulmonary disease. Eur Respir J 1997; 10: 2794-2800.

3 Curkendall SM, DeLuise C, Jones JK, et al. Cardiovascular disease in patients with chronic obstructive pulmonary disease, Saskatchewan Canada: cardiovascular disease in COPD patients. Ann Epidemiol 2006; 16: 63-70.

4 Anthonisen NR, Connett JE, Enright PL, et al. Hospitalizations and mortality in the Lung Health Study. Am J Respir Crit Care Med 2002; 166: 333-339.

5 Halpin DM, Decramer M, Celli B, et al. Risk of nonlower respiratory serious adverse events following COPD exacerbations in the 4-year UPLIFT $^{\circ}$ trial. Lung 2011; 189: 261-268.

6 Ghoorah K, De Soyza A, Kunadian V. Increased cardiovascular risk in patients with chronic obstructive pulmonary disease and the potential mechanisms linking the two conditions: a review. Cardiol Rev 2013; 21: 196-202.

7 Williams MC, Murchison JT, Edwards LD, et al. Coronary artery calcification is increased in patients with COPD and associated with increased morbidity and mortality. Thorax 2014; 69: 718-723.

8 Maclay JD, McAllister DA, Johnston S, et al. Increased platelet activation in patients with stable and acute exacerbation of COPD. Thorax 2011; 66: 769-774.

9 Zheng SL, Roddick AJ. Association of aspirin use for primary prevention with cardiovascular events and bleeding events: a systematic review and meta-analysis. JAMA 2019; 321: 277-287.

10 Bhatt DL, Fox K, Harrington RA, et al. Rationale, design and baseline characteristics of the effect of ticagrelor on health outcomes in diabetes mellitus patients intervention study. Clin Cardiol 2019; 42: 498-505.

11 Kunadian V, Chan D, Ali $\mathrm{H}$, et al. Antiplatelet therapy in the primary prevention of cardiovascular disease in patients with chronic obstructive pulmonary disease: protocol of a randomised controlled proof-of-concept trial (APPLE COPD-ICON 2). BMJ Open 2018; 8: e020713.

12 Mehran R, Rao SV, Bhatt DL, et al. Standardized bleeding definitions for cardiovascular clinical trials: a consensus report from the Bleeding Academic Research Consortium. Circulation 2011; 123: 2736-2747.

13 Jung SH. Randomized phase II trials with a prospective control. Stat Med 2008; 27: 568-583.

14 Perl L, Zemer-Wassercug N, Rechavia E, et al. Comparison of platelet inhibition by prasugrel versus ticagrelor over time in patients with acute myocardial infarction. J Thromb Thrombolysis 2015; 39: 1-7.

15 Divo M, Cote C, de Torres JP, et al. Comorbidities and risk of mortality in patients with chronic obstructive pulmonary disease. Am J Respir Crit Care Med 2012; 186: 155-161.

16 Harrison MT, Short P, Williamson PA, et al. Thrombocytosis is associated with increased short and long term mortality after exacerbation of chronic obstructive pulmonary disease: a role for antiplatelet therapy? Thorax 2014 69: 609-615.

17 Huber MB, Wacker ME, Vogelmeier CF, et al. Excess costs of comorbidities in chronic obstructive pulmonary disease: a systematic review. PLoS One 2015; 10: e0123292.

18 Jakl M, Sevcik R, Fatorova I, et al. High on-treatment platelet reactivity: risk factors and 5-year outcomes in patients with acute myocardial infarction. Anatol J Cardiol 2017; 17: 113-118.

19 Campo G, Pavasini R, Pollina A, et al. On-treatment platelet reactivity in patients with chronic obstructive pulmonary disease undergoing percutaneous coronary intervention. Thorax 2014; 69: 80-81.

20 Bergmeijer TO, Janssen PWA, van Oevelen M, et al. Incidence and causes for early ticagrelor discontinuation: a "real-world" Dutch registry experience. Cardiology 2017; 138: 164-168.

21 Koziński M, Ostrowska M, Adamski $\mathrm{P}$, et al. Which platelet function test best reflects the in vivo plasma concentrations of ticagrelor and its active metabolite? The HARMONIC study. Thromb Haemost 2016; 116: $1140-1149$.

22 Mingant F, Didier R, Gilard M, et al. Comparison of four methods to assess high-on platelet reactivity under P2Y12 receptor inhibitor. Platelets 2018; 29: 257-264.

23 Legrand V, Cuisset T, Chenu $\mathrm{P}$, et al. Platelet reactivity and cardiovascular events after percutaneous coronary intervention in patients with stable coronary artery disease: the Stent Thrombosis In Belgium (STIB) trial. EuroIntervention 2014; 10: 204-211.

24 Storey RF, Becker RC, Harrington RA, et al. Characterization of dyspnoea in PLATO study patients treated with ticagrelor or clopidogrel and its association with clinical outcomes. Eur Heart J 2011; 32: 2945-2953.

25 Andell P, James SK, Cannon CP, et al. Ticagrelor versus clopidogrel in patients with acute coronary syndromes and chronic obstructive pulmonary disease: an analysis from the Platelet Inhibition and Patient Outcomes (PLATO) trial. J Am Heart Assoc 2015; 4: e002490.

26 Butler K, Maya J, Teng R. Effect of ticagrelor on pulmonary function in healthy elderly volunteers and asthma or chronic obstructive pulmonary disease patients. Curr Med Res Opin 2013; 29: 569-577.

27 Kawase Ishihara K, Kokubo Y, Yokota C, et al. Effect of plasma fibrinogen, high-sensitive c-reactive protein, and cigarette smoking on carotid atherosclerosis: the Suita study. J Stroke Cerebrovasc Dis 2015; 24: 2385-2389.

28 Sin DD, Man SF. Why are patients with chronic obstructive pulmonary disease at increased risk of cardiovascular diseases? The potential role of systemic inflammation in chronic obstructive pulmonary disease. Circulation 2003 107: 1514-1519. 
29 Andreas S, Anker SD, Scanlon PD, et al. Neurohumoral activation as a link to systemic manifestations of chronic lung disease. Chest 2005; 128: 3618-3624.

30 Campo G, Vieceli Dalla Sega F, Pavasini R, et al. Biological effects of ticagrelor over clopidogrel in patients with stable coronary artery disease and chronic obstructive pulmonary disease. Thromb Haemost 2017; 117: 1208-1216. 